\title{
The Effect of Massage on Salivary Secretory Iga Level in Preterm Infants
}

\author{
(1) Armin Karamian1, (1) Majid Firouzi2, (1) Roshanak Modiri1, (1) Amin Karamian³ \\ 'Lorestan University of Medical Sciences, School of Medicine, Khoramabad, Iran \\ 2Lorestan University of Medical Sciences, Faculty of Medicine, Department of Pediatrics, Khoramabad, Iran \\ 3Shahid Beheshti University School of Medicine, Department of Biology and Anatomical Sciences, Tehran, Iran
}

\begin{abstract}
Aim: Secretory Immunoglobulin A ( $\lg A$ ) is one of the most important components of the immune system and has a prominent role in preventing infections that are transmitted through the gastrointestinal tract and respiratory mucosa. The primary objective of this study was to evaluate the effect of massage on the salivary slgA level in pre-term infants and the secondary objective was to assess the effect of massage on neonatal weight gain.
\end{abstract}

Materials and Methods: This study was performed on 150 preterm infants weighing between 1,500 gm and 2,000 gm who were hospitalized in 3 main medical centers of Khorramabad city, Iran between March 2018 and March 2019. The 3 major selected centers were Asalian, Shahid Madani and Shahid Rahimi Hospitals. All infants were randomly allocated to either the intervention $(n=75)$ or the control $(n=75)$ group. Due to our criteria, 18 patients were excluded from this study. In total, 132 infants were included in this study. The initial samples of slgA were obtained from the saliva of the neonates in both groups on the second day after birth and when acute symptoms of respiratory distress syndrome had resolved. The second samples were collected on the $14^{\text {th }}$ day after birth. During this period, neonates of the intervention group were massaged properly using the Field technique for 15 minutes, three times a day by a trained Neonatal Intensive Care Unit nurse for 12 consecutive days. It should be noted that due to the effect of breast milk on the neonates' slgA levels, we selected all subjects from those who were not fed with breast milk for some reason, but were fed with formula instead.

Results: According to our findings, a significant difference was observed between the mean weight of the infants between the second and $14^{\text {th }}$ days after birth in both the intervention and control groups $(p<0.001)$. However, in comparison, there was no significant difference between the mean weight changes of the infants in both groups $(p=0.845)$. Moreover, there was a significant difference between the mean slgA level changes between the second and $14^{\text {th }}$ days after birth in both groups $(p<0.001)$, but these changes were more significant in the intervention group compared to the control group $(p<0.001)$.

Conclusion: These results showed that massage therapy in neonates can increase the level of slgA which plays an important part in the infant's immune system. However, this had no positive effect on the weight gain process. It is recommended that massage therapy be used as a safe auxiliary therapeutic procedure for preterm infants and that mothers can learn and perform this technique as a simple way to boost the immune system of their infants.

Keywords: Massage, salivary secretory IgA, preterm infants, immune system, preterm, weight gain 


\section{Introduction}

Massage therapy is an interesting topic in neonatal medicine and many studies have been conducted over the last decade. New studies support the efficacy of massage therapy as a complementary therapeutic method in neonatal medicine (1). Touching and stroking infants during massage stimulate nerve pathways and speed up myelination of the brain and nervous system through increased hypothalamic activity followed by increased somatotropin levels. In animal studies, the lack of skin and tactile stimuli reduces the growth of all organs, and when the animals return to normal tactile stimulation, this defect is eliminated, which highlights the beneficial effects of massage on growth hormone levels (2). Massage inside the infant's mouth increases the levels of gastrointestinal hormones such as insulin and gastrin, which highlights the fact that skin stimulation and massage in the same way will increase similar hormones. Studies on neonatal insulin and glucose levels have shown that massage in preterm infants will increase insulin and IGF-1 levels (1). In the field of pain management in children, it has been mentioned that massage by parents during sleep is useful for reducing chronic and myofascial pain (3). It is believed that, like breastfeeding by the mother, massage by the father will facilitate proper bonding between the father and infant. The mother as a massager reduces the level of stress hormones in the mother's body and significantly reduces the rate of postpartum depression and anxiety $(1,4)$.

Neonatal Intensive Care Units (NICUs) are full of stress and massage therapy can lessen the level of stress hormones in preterm infants, as well as improve the developmental status of low-birthweight neonates (5-7). Immunoglobulins (Ig) are components of the humoral immune system that are produced in response to internal and external antigens. Each antibody molecule has an overall symmetrical structure consisting of four polypeptide chains, two identical light chains and two identical heavy chains. Antibody molecules are classified into different classes, subclasses, types and subtypes based on differences in the structures of the heavy and light chain constant regions. There are five main classes of Igs: IgG, IgM, IgA, IgD, and IgE-some of which include a number of distinct subclasses (8).

The placenta selectively blocks various substances to prevent transmission from the mother to the fetus. Of all the antibodies, only IgG can cross the placental barrier. IgA and IgM are blocked from crossing the placenta and so their levels in infancy confirm their production by the infant itself $(9,10)$.
IgA comprises monomeric and polymeric IgA of two subtypes, $\lg \mathrm{A}_{1}$ and $\lg \mathrm{A}_{2}$. In adults, $\lg \mathrm{A}_{1}$ is the main subtype in serum but, in the mucosa, $\lg \mathrm{A}_{2}$ production is higher than $\lg \mathrm{A}_{1}$ (11). Serum IgA is mainly monomeric while secretory IgA is dimeric and is joined by the J chain and the secretory part. IgA is often secreted by the secretory cells of the breast as a dimer. IgA is the main antibody found in many body secretions, including tears, saliva, respiratory and intestinal secretions, mucous and milk (12). IgA is the most important Ig in breast milk and it protects the mucosal surfaces against foreign pathogens until the infant progressively is able to make its own IgA, which sometimes takes weeks to months after birth. It can also protect infants from diseases which are transmitted via the mucosa of the gastrointestinal tract and respiratory system $(9,10,13)$.

In infants, due to the underdeveloped humoral and cellular immune systems, the risk of infection is significantly increased compared to other age groups. Therefore, in infants, limited infections are potentially more likely to become systemic and severe infections (14). Akimoto et al. (15) showed that 12 months of exercise could significantly increase both the concentration and secretion rate of secretory $\lg A$ ( $\operatorname{sg} A$ ) in elderly subjects. The concentration of salivary IgA was significantly increased in subjects who received four relaxation methods including massage therapy, compared with a touch control group (16). One analysis found that receiving massages is an effective method to recover from temporary immunosuppression state induced by heavy exercise and to enhance levels of $\operatorname{slg} A(17)$.

In the current study, we aimed to investigate the effect of neonatal massage on increasing the level of slgA in the saliva of preterm infants who were admitted to Asalian, Shahid Madani and Shahid Rahimi Hospitals in Khorramabad city, Iran between March 2018 and March 2019.

\section{Materials and Methods}

This randomized clinical trial was conducted on 150 preterm infants who were admitted to NICUs in the three main hospitals in Khorramabad city, Iran between March 2018 and March 2019. Initially, the sample size was calculated to be 63 for each group. However, to ensure the adequacy of the participants, 75 infants were allocated to each group. Infants were selected from formula-fed infants because of the possible effect of breastfeeding on slgA levels. The participants were randomly divided into intervention and control groups. The inclusion criteria were as follows: preterm infants with gestational age $<37$ weeks, weighing 
between 1,500 and 2,000 gm, formula-fed, an absence of congenital infections, no congenital anomalies, an having no prohibition of massage (e.g. skin lesions, hematoma or dislocation of limbs).

Infants were excluded from this study if any of the following criteria applied: evidence in favor of infection (for example, positive blood culture or positive (SF culture), a requirement for mechanical ventilation, a history of seizure or an occurrence of seizure during the study, a history of immunodeficiency among first-degree relatives, a requirement for blood transfusion, transferal of the infant to another hospital for any reason, the presence of any congenital anomalies, a history of maternal illness during the pregnancy period prior to delivery such as hepatitis B or syphilis (based on screening tests), a requirement for surgery in the course of the study, discharge from the hospital, dissatisfaction of the parents with respect to continuing the study and receiving some medications for instance, antibiotics and intravenous Ig.

Written consent was obtained from the parents before enrollment in the study. Hundred-fifty infants were randomly divided into 2 groups: The control group $(n=75)$ and the intervention group $(n=75)$. Of these, 18 cases were excluded ( 7 infants from the intervention and 11 infants from the control group) and 132 infants remained until the completion of the study (68 infants in the intervention and 64 infants in the control group). The major reason of exclusion was the lack of sufficient saliva ( 3 infants in the intervention and 5 infants in the control group). Other reasons for exclusion were as follows: The parent's unwillingness to continue the study ( 3 infants from the intervention and 4 infants from the control group) and 3 infants died during the study (one infant in the intervention and 2 infants in the control group).

In the intervention group, 41 (60.3\%) and 27 (39.7\%) of the cases were male and female, respectively. In the control group, 31 (48.4\%) and $33(51.6 \%)$ of the subjects were male and female, respectively. In terms of gender, both groups were similar $(p=0.22)$.

Only the researchers were aware of infant's groups (intervention or control) and the laboratory was blind to which group they were in. The neonates in both groups were administered standard ward care during the course of this study. Based on standards for NICUs, the room temperature was between $2-26^{\circ} \mathrm{C}$.

We performed the massage based on the Field technique. Massaging was gentle using the ventral surface of both hands with gentle pressure and it was performed by a trained NICU nurse who played no other role in the study and she was also blind to the hypothesis of the study. Before starting the massage, her hands were washed and warmed. Sunflower oil was used as a lubricant. The infants were placed in a quiet environment under a radiant warmer. The infants of the intervention group received massages for 15 minutes, three times per day, for 12 consecutive days. Each massage session consists of three phases of 5 minutes. The infants received their massages one hour after being fed. During the first and third phase of the massage therapy, the neonate was placed in a prone position, and was massaged for five one-minute periods (five seconds per stroking motion) over each region in the following sequence: A) 12 stroking movements from the top of the head to the neck downwards and then reversed; B) 12 stroking movements from the neck to the shoulders and then reversed; C) 12 stroking movements from the upper back down to the waist and then reversed; D) 12 stroking movements from the thighs down to the ankles and the reversed; E) 12 stroking movements from the shoulder to the hand and then reversed on both arms. In the second phase, the infant was put in a supine position and six flexion/extension moves were performed on the limbs in the following order: each arm, each leg and finally both legs together. Each flexion/extension movement lasted approximately 10 seconds. The control group did not receive massage therapy during the study.

In both groups, the initial and second saliva samples were obtained using a sterile infant mucus extractor from the mouth of the neonates. The initial and second samples were collected on the second and $14^{\text {th }}$ day after birth, respectively. The salivary slgA level was measured using an IgA saliva ELISA kit (DiaMetra, Perugia, Italy) according to the manufacturer's protocol.

In both groups, the weight of the neonates was also measured on the second and $14^{\text {th }}$ day after birth. This study did not interfere with the treatment of the infants.

All phases of this study and consent forms were approved by the Ethics Committee of Lorestan University of Medical Sciences (LUMS.REC.1396.232) and the registration ID of this study in Iranian Registry of Clinical Trials is IRCT20171225038056N1.

\section{Statistical Analysis}

Data were expressed as means \pm standard deviation. After determining the measures of central tendencies and measures of dispersion, independent t-test and paired t-test were used to analyze the data. All results were 
analyzed using IBM SPSS for Windows, Version 21.0 (IBM Corp., Armonk, NY, USA). A p-value $<0.05$ was considered statistically significant for all analyses.

\section{Results}

According to the findings, the mean weight of the infants in the control group was 1,706.95 $\pm 146.07 \mathrm{gm}$ and $2,051.64 \pm 157.41 \mathrm{gm}$ on the second and $14^{\text {th }}$ day after birth, respectively. There was also a significant difference between the mean weight of the infants in the control group on the second and $14^{\text {th }}$ day after birth $(p<0.001)$ (Figure 1$)$. The mean weight of the infants in the intervention group was $1,684.41 \pm 136.4 \mathrm{gm}$ on the second day and 2,032.42 \pm 145.49 $\mathrm{gm}$ on the $14^{\text {th }}$ day after birth. There was a significant difference between the mean weight of the infants in the intervention group on the second and $14^{\text {th }}$ day after birth $(p<0.001)$ (Figure 1).

According to the results, the mean weight changes showed no significant difference between the intervention and control groups on the second or $14^{\text {th }}$ day after birth $(p=0.845)$ (Figure 2).

The mean slgA levels in the control group on the second and $14^{\text {th }}$ day were $8.25 \pm 3.76(\mu \mathrm{g} / \mathrm{dL})$ and $9.68 \pm 3.67(\mu \mathrm{g} /$ $\mathrm{dL})$, respectively, and there was also a significant difference between the mean slgA level on the second and $14^{\text {th }}$ day $(p<0.001)$ (Figure 3). Moreover, the mean slgA levels in the intervention group on the second and $14^{\text {th }}$ day after birth were $9.71 \pm 4.52(\mu \mathrm{g} / \mathrm{dL})$ and $17.8 \pm 6.4(\mu \mathrm{g} / \mathrm{dL})$, respectively, and we found a significant difference between the mean slgA levels before and after massage therapy $(p<0.001)$ (Figure 3).

The results of our study demonstrated that the mean slgA level changed between the intervention and control

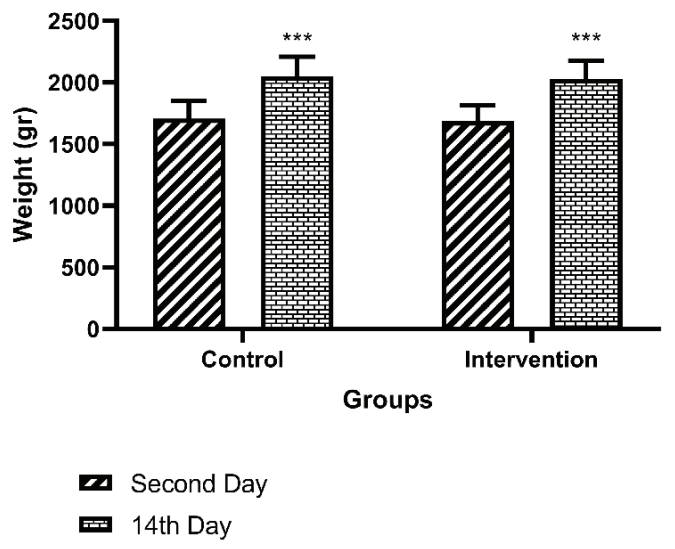

Figure 1. Comparison of mean weight changes between second and $14^{\text {th }}$ day after birth in the intervention and control groups groups on the second and $14^{\text {th }}$ day after birth and there was a significant difference between the two groups $(p<0.001)$ (Figure 4).

\section{Discussion}

IgA is one of the most important lgs in the human body and it protects infants against those diseases that are transmitted via the mucosa of the gastrointestinal tract and respiratory system (13). Massage therapy is a new subject in neonatal medicine and new studies support the efficacy of massage as a safe and complementary therapeutic method in the field of neonates (1).

In the present study, for the first time, the effect of massage therapy on salivary slgA levels in preterm infants was investigated. Our findings in this research provide

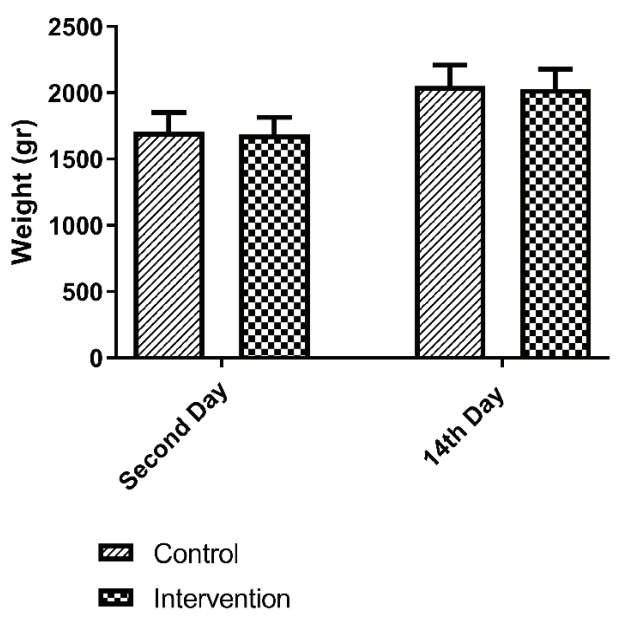

Figure 2. Comparison of mean weight changes between the intervention and control groups on the second and $14^{\text {th }}$ day after birth

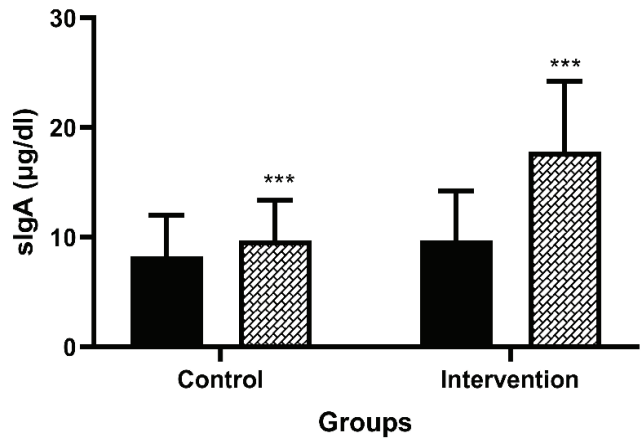

$$
\begin{aligned}
& \text { Second Day } \\
& \text { 14th Day }
\end{aligned}
$$

Figure 3. Comparison of mean slgA level changes between the intervention and control groups on the second and $14^{\text {th }}$ day after birth slgA: Secretory immunoglobulin A 


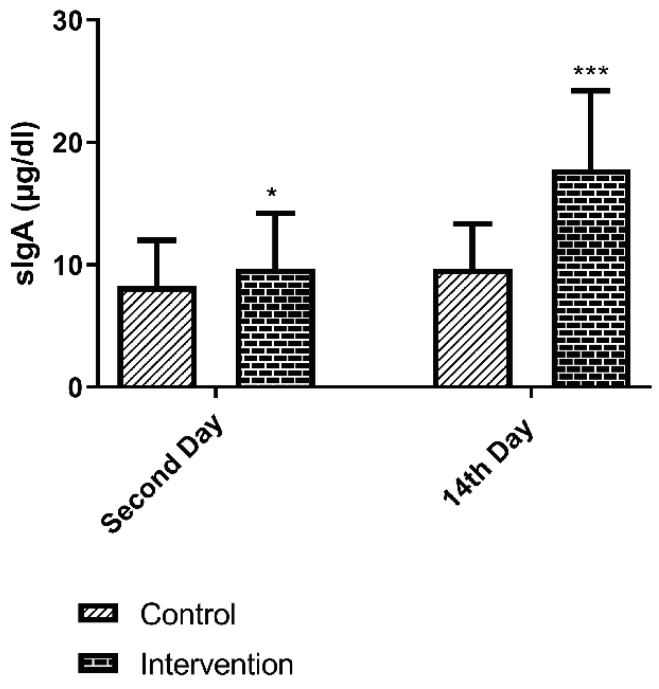

Figure 4. Comparison of mean slgA level changes between the intervention and control groups on the second and 14th day after birth slgA: Secretory immunoglobulin A

evidence indicating that the slgA levels was significantly higher on the $14^{\text {th }}$ day in the intervention group than in the control group, but we could not observe an increase regarding the mean weight parameter.

Some previous studies have addressed the effects of massage on improving bone density $(6,18)$, neurodevelopmental status in preterm infants $(19,20)$, bilirubin levels of infants with jaundice $(21,22)$, sleep quality in infants with low birth weight (23) and reducing the length of hospital stays (24).

Our study showed that weight gain was not significantly different between those infants who received massages and those who did not. This result was contrary to previous studies (7,25-29).

A study performed by Scafidi et al. (29) on preterm infants demonstrated that tactile/kinesthetic stimulation can improve weight gain and sleep behavior in low-birthweight infants. In another study, they also showed that massage can accelerate the process of discharging lowbirth-weight infants from the hospital by 6 to 10 days (30). Research conducted on infants aged between 32 and 37 weeks found that massage has a significant effect on improving mental-behavior status (20). Another study evaluated the effect of massage on salivary cortisol levels in preterm infants and showed that massage therapy can reduce the level of cortisol as a stress indicator in saliva (31). The results of another study demonstrated that the
Field technique massage and gentle human touch can also decrease urine cortisol levels in preterm infants (5).

Ang et al. (32) investigated the effect of massage therapy on the immune system of preterm infants and they found that this method could be associated with higher NK cell cytotoxicity.

We believe that more subjects should be included in future studies and further studies are needed on this new topic in neonatology. Finally, it is recommended that massage therapy be performed by parents or trained nurses as a complementary therapeutic method for infants.

\section{Conclusion}

According to the results of our study, the slgA levels in preterm infants who received massage for 12 consecutive days was significantly higher compared to a control group who did not receive any intervention. Furthermore, at the end of the study, there was no significant difference in weight gain between the intervention and control groups. Massage therapy can be used as a safe complementary practice for infants and it can also promote bonding between the mother and infant. Our observations provide new information about the effect of massage therapy on boosting the mucosal immune system in preterm infants. However, further studies can be carried out to investigate the use of massage therapy on more samples over a longer period of time in order to clarify the true effects of such complementary methods on the immune system and on the outcomes of neonatal infections.

\section{Ethics}

Ethics Committee Approval: All phases of the study and consent forms were approved by the Ethics Committee of Lorestan University of Medical Sciences (LUMS. REC.1396.232) and the registration ID of this study in Iranian Registry of Clinical Trials is IRCT20171225038056N1.

Informed Consent: A written consent was obtained from parents before enrollment in the study.

Peer-review: Externally and internally peer-reviewed.

\section{Authorship Contributions}

Concept: M.F., Design: Ar.K., Data Collection or Processing: Ar.K., Analysis or Interpretation: R.M., A.K., Literature Search: M.F., Writing: Ar.K., R.M., A.K.

Conflict of Interest: The authors declare that they have no conflict of interest.

Financial Disclosure: The authors declared that this study received no financial support. 


\section{References}

1. Martin RJ, Fanaroff AA, Walsh MC. Fanaroff and Martin's Neonatal-Perinatal Medicine: Diseases of the Fetus and Infant, Vol 2, 10 ed2014. p. 327-50.

2. Gardner SL, Carter BS, Enzman-Hines M, Hernandez JA. Merenstein \& Gardner's Handbook of Neonatal Intensive Care, 7 ed2010. p. 245-62.

3. Kliegman RM, Stanton B, St. Geme JW, Schor NF. Nelson Textbook of Pediatrics, Vol 1, 20 ed2015. p. 442.

4. Tricia Lacy Gomella MDC, Fabien G. Eyal. Neonatology: Management, Procedures, On-Call Problems, Diseases, and Drugs, 7 ed2013. p. 217-8.

5. Asadollahi M, Jabraeili M, Mahallei $M$, Asgari Jafarabadi $M$, Ebrahimi S. Effects of Gentle Human Touch and Field Massage on Urine Cortisol Level in Premature Infants: A Randomized, Controlled Clinical Trial. J Caring Sci 2016; 5:187-94.

6. Stalnaker KA, Poskey GA. Osteopenia of Prematurity: Does Physical Activity Improve Bone Mineralization in Preterm Infants? Neonatal Netw 2016; 35:95-104.

7. Vickers A, Ohlsson A, Lacy IB, Horsley A. Massage for promoting growth and development of preterm and/or low birth-weight infants. Cochrane Database Syst Rev 2004;2004:Cd000390.

8. Schroeder Jr HW, Cavacini L. Structure and function of immunoglobulins. The Journal of allergy and clinical immunology 2010; 125:S41-S52.

9. Quigley J. The role of oral immunoglobulins in systemic and intestinal immunity of neonatal calves. Ames, IA, lowa State University 2004.

10. Ravivarma KR, Babar ST, Master J, Bapat IP, Baxi AJ. Immunoglobulins in newborns: differential study of premature and full term infants. J Postgrad Med 1979; 25:97-101.

11. Brandtzaeg P, Farstad IN, Johansen FE, Morton HC, Norderhaug IN, Yamanaka T. The B-cell system of human mucosae and exocrine glands. Immunol Rev 1999; 171:45-87.

12. Goldman AS, ChhedaS, Keeney SE, Schmalstieg FC. Immunology of Human Milk and Host Immunity. Fetal and Neonatal Physiology 2011; 1690-701.

13. Pineda-Martínez S, Hernández-Islas JL, Escobedo-Torres MP, et al. Immunoglobulin Concentrations in Plasma and Saliva During the Neonatal Period. Pediatr Neonatol 2016; 57:213-8.

14. Mhairi G. MacDonald MMKS, Martha D. Mullett. Avery's Neonatology: Pathophysiology and Management of the Newborn2015. p. 506-7.

15. Akimoto $T$, Kumai $Y$, Akama $T$, et al. Effects of 12 months of exercise training on salivary secretory IgA levels in elderly subjects. Br I Sports Med 2003; 37:76-9.

16. Green RG, Green ML. Relaxation increases salivary immunoglobulin A. Psychological Reports 1987; 61:623-9.

17. Arroyo-Morales $M$, Olea $N$, Ruíz $C$, et al. Massage after exercise--responses of immunologic and endocrine markers: a randomized single-blind placebo-controlled study. I Strength Cond Res 2009; 23:638-44.
18. Haley S, Beachy I, Ivaska KK, Slater H, Smith S, Moyer-Mileur L). Tactile/kinesthetic stimulation (TKS) increases tibial speed of sound and urinary osteocalcin (U-MidOC and unOC) in premature infants (29-32weeks PMA). Bone 2012; 51:661-6.

19. Lai MM, D'Acunto G, Guzzetta A, et al. PREMM: preterm early massage by the mother: protocol of a randomised controlled trial of massage therapy in very preterm infants. BMC Pediatrics 2016; 16:146.

20. Rajaram R, Vizhi DM, Kumar V. Effects of early neonatal massage with therapeutic positioning in preterm and low birth weight babies on neurobehavioral and neurodevelopmental status. Int J Pharm Bio Sci 2017; 8.

21. Dalili H, Sheikhi S, Shariat M, Haghnazarian E. Effects of baby massage on neonatal jaundice in healthy Iranian infants: A pilot study. Infant Behav Dev 2016; 42:22-6.

22. Lin $\mathrm{CH}$, Yang HC, Cheng CS, Yen CE. Effects of infant massage on jaundiced neonates undergoing phototherapy. Ital I Pediatr 2015; 41:94.

23. Kelmanson IA, Adulas El. Massage therapy and sleep behaviour in infants born with low birth weight. Complement Ther Clin Pract 2006; 12:200-5.

24. Mendes EW, Procianoy RS. Massage therapy reduces hospital stay and occurrence of late-onset sepsis in very preterm neonates. J Perinatol 2008; 28:815-20.

25. Diego MA, Field T, Hernandez-Reif M, Deeds O, Ascencio A, Begert G. Preterm infant massage elicits consistent increases in vagal activity and gastric motility that are associated with greater weight gain. Acta Paediatr 2007; 96:1588-91.

26. Field T, Diego MA, Hernandez-Reif M, Deeds O, Figuereido $B$. Moderate versus light pressure massage therapy leads to greater weight gain in preterm infants. Infant Behav Dev 2006; 29:574-8.

27. Jabraeile M, Rasooly AS, Farshi MR, Malakouti J. Effect of olive oil massage on weight gain in preterm infants: $A$ randomized controlled clinical trial. Niger Med J 2016; 57:160-3.

28. Rad Z, Haghshenas M, Javadian Y, Hajiahmadi M, Kazemian F. The effect of massage on weight gain in very low birth weight neonates. J Clin Neonatol 2016; 5:96-9.

29. Scafidi FA, Field TM, Schanberg SM, et al. Effects of tactile/ kinesthetic stimulation on the clinical course and sleep/wake behavior of preterm neonates. Infant Behavior and Development 1986; 9:91-105.

30. Scafidi FA, Field TM, Schanberg SM, et al. Massage stimulates growth in preterm infants: A replication. Infant Behavior and Development 1990; 13:167-88.

31. Asmarani R, Irwanto I, Suryawan A, Irmawati M, Utomo M. Effect of Massage on Salivary Cortisol Level in Preterm Neonates. Iranian Journal of Neonatology IJN 2020; 11:12-6.

32. Ang JY, Lua JL, Mathur A, et al. A randomized placebo-controlled trial of massage therapy on the immune system of preterm infants. Pediatrics 2012; 130:e1549-58. 